\title{
Molecular Properties of Astaxanthin in Water/Ethanol Solutions from Computer Simulations
}

\author{
Khadga Jung Karki, ${ }^{\dagger}$ Susruta Samanta ${ }^{\ddagger}{ }^{\dagger}$ and Danilo Roccatano*, \\ †Jacobs University Bremen, Campus Ring 8 28759, Bremen Germany \\ $\ddagger$ Department of Physics, University of Cagliari, S.P. Monserrato-Sestu Km 0.700, \\ I-09042, Monserrato (CA), Italy \\ ฯ School of Mathematics and Physics, University of Lincoln. Brayford Pool, LN6 7TS, \\ Lincoln, $U K$ \\ E-mail: droccatano@lincoln.ac.uk \\ Phone: $+44(0) 1522835868$
}

\begin{abstract}
Astaxanthin (AXT) is a reference model of xanthophyll carotenoids, which is used in medicine and food industry, and has potential applications in nanotechnology. Because of its importance, there is a great interest in understanding its molecular properties and aggregation mechanism in water and mixed solvents. In this paper, we report a novel model of AXT for molecular dynamics simulation. The model is used to estimate different properties of the molecule in pure solutions and in water/ethanol mixtures.
\end{abstract}


The calculated diffusion coefficients of AXT in pure water and ethanol are $(3.22 \pm 0.01) \times 10^{-6} \mathrm{~cm}^{2} \mathrm{~s}^{-1}$ and $(2.7 \pm 0.4) \times 10^{-6} \mathrm{~cm}^{2} \mathrm{~s}^{-1}$, respectively. Our simulations also show that the content of water plays a clear effect on the morphology of the AXT aggregation in water/ethanol mixture. In up to $75 \%(\mathrm{v} / \mathrm{v})$ water concentration, loosely connected network of dimers and trimers, and two-dimensional array structures are observed. At higher water concentrations, AXT molecules form more compact three-dimensional structures that are preferentially solvated by the ethanol molecules. The ethanol preferential binding and the formation of a well connected hydrogen bonding network on these AXT clusters, suggest that such preferential solvation can play an important role in controlling the aggregate structure.

\section{Introduction}

Astaxanthin (AXT), (3R,3R')- dihydroxy- $\beta$-carotene-4,4'-dione, is a xanthophyll carotenoid, which is commonly found in aquatic animals, such as the Antarctic krill (Euphausia superba). ${ }^{1}$ In the exoskeleton of lobsters, AXT is associated with the protein crustacyanin that produces the typical blue colour of the an-

imal. AXT is also present in large quantities in single green microalgal cell (Haematococcus pluvialis) dissolved in oil droplets as shown by the recent x-ray micro-tomographic of these droplets in the cells. ${ }^{2}$ The molecular structure of AXT consists of a polyene chain terminated with two symmetrically functionalized cyclohexen groups (see Fig. 1). Similar to other carotenoids, AXT is also a powerful antioxidant, which acts as a free-radical scavenger and an immuno-modulator. ${ }^{3,4}$ These properties have beneficial healing effects against degenerative diseases related to oxidative stress/damage, neurodegenerative diseases and cancers. ${ }^{4-6}$

Carotenoids, as found in light harvesting complexes, form large aggregates 
with functional properties. Their photophysical properties and functionality are influenced by the surrounding environment. In particular, their photophysical properties change dramatically with the environment due to the formation of different types of aggregates. ${ }^{7,8}$ As many other carotenoids and chlorophylls, ${ }^{9-11}$ AXT also forms aggregates when dissolved in hydrated polar solvents. ${ }^{8,12}$ The water concentration in these mixtures influences the morphology of the aggregates, and, consequently, their properties. In particular, spectroscopic analysis have shown that their absorption spectra can be blue shifted as well as red shifted compared to that of the isolated monomers depending on the aggregation conditions. ${ }^{7,8,12-14}$ The blue shift is attributed to the formation of $H$-aggregates and the red shift is attributed to the formation of $J$-aggregates. The $H$-aggregates formed by these molecules when deposited on surfaces or arranged at interfaces, hold promise as photoactive species in dye-sensitized solar cells. ${ }^{15}$ However, due to the lack of suitable experimental methods, the aggregation mechanism as well as the effect of the environment on the three-dimensional structure of both types of aggregates is not yet fully understood. ${ }^{16}$ Theoretical approaches, such as molecular dynamics (MD) simulations, can provide accurate atomistic details on their structural and dynamical properties in solution. Still, to the best of our knowledge, there is a paucity of MD simulation studies on this molecule from the literature. In order to advance the understanding of the structural and dynamic properties of AXT in solution, in this paper we will present a new accurate model of AXT for MD simulations based on the OPLS force field. ${ }^{17}$ The model was tested for transport properties of the single AXT molecule in pure water and ethanol, and for the aggregation properties in different ethanol/water solutions. In this paper, we have focused on the qualitative analysis of aggregation dynamics and on morphology of aggregates at different ethanol/water mixtures concentra- 
tions. These properties and the aggregation mechanism have been discussed and compared with the available experimental data.

\section{Material and Methods}

Force field parameters. The starting structure of the molecule without hydrogen atoms were obtained from the protein data bank (www.pdb.org, PDB code: AXT). This is an all-trans structure with the two asymmetric carbon atoms in $R$ configuration. After adding hydrogen atoms, the structure was optimised in vacuum using Density Functional Theory method with the B3LYP functional and the $6-31 \mathrm{G}^{* *}$ basis set. The Gaussian 09 program $^{20}$ was used for the calculations. The optimised atomic coordinates of the molecule (showed in Fig.1) are reported in Table S1 of the SI.

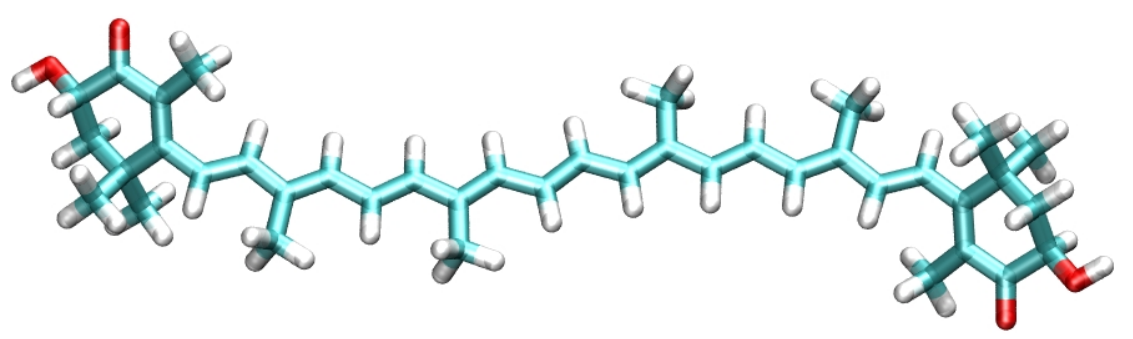

Figure 1: Optimized structure of AXT.

The CHELPG procedure ${ }^{21}$ was used to calculate the partial charges on the atoms of the optimised structure by fitting the electrostatic potential generated by the electron density. The fitting procedure was constrained to reproduce the calculated dipole moment of the molecule. The QM calculated partial charges were further adjusted by averaging the values of symmetry related atoms (see Table $3 \mathrm{~S}$ in SI).

The geometric reference parameters for the bonded interactions were taken 
from the QM optimised structure whereas their force constants were adapted from the OPLS force field library. ${ }^{17,22-25}$ For the ethanol and water, the united atom OPLS model, ${ }^{26}$ and the SPC/E model ${ }^{27}$ have been used, respectively.

Molecular Dynamics Simulations. The composition of the simulated systems are shown in Table 1. All MD simulations and analysis were performed using the GROMACS package (version 4.5.5). ${ }^{28}$ The systems were prepared by either centering or randomly distributing the AXT molecule(s) in a cubic box. The remaining empty space was then filled by stacking an optimized small box of solvent molecules and by removing solvent molecules overlapping with atoms of the AXT molecules. The co-solvent concentrations in the mixture were adjusted by adding or removing the required amount of molecules. In order to compare with the available experimental data, the solvent concentrations were calculated as percentage volume/volume using the experimental molar volumes $\bar{V}_{w}=2.99 \times$ $10^{-2}$ and $\bar{V}_{e}=8.97 \times 10^{-2} \mathrm{~nm}^{3} / \mathrm{mol}$ for water and ethanol, respectively.

During the simulations, the temperature and the pressure were kept constant at $300 \mathrm{~K}$ and 1 bar using the Nose-Hoover ${ }^{29}$ thermostat and the ParrinelloRahman ${ }^{30}$ barostat, respectively. The coupling time for the temperature and the pressure were set to 0.2 and $1.0 \mathrm{ps}$, respectively. The isothermal compressibility was set to $4.5 \times 10^{-5}$ bar $^{-1}$ for all the systems. The SHAKE algorithm ${ }^{31}$ was used to constrain all bond lengths. A 1.0-1.2 nm switched cutoff radius was used for the calculation of the Lennard-Jones interactions. The electrostatic interactions were calculated using the PME method ${ }^{32}$ of the $4^{\text {th }}$ order with the Fourier spacing of $0.12 \mathrm{~nm}$ and dielectric permittivity $\epsilon_{r}=1$. The short-range cutoff for the neighbor list was set to $1.4 \mathrm{~nm}$. All atoms were given an initial velocity obtained from a Maxwell-Bolztmann distribution at $300 \mathrm{~K}$. An integration time step of 2 fs was used. All the systems were equilibrated for 500 ps to bring them to a 
constant density. After the equilibration, production runs of $200 \mathrm{~ns}$, for the single $\mathrm{AXT}$ in pure solvents, and $50 \mathrm{~ns}$, for $10 \mathrm{AXT}$ in the mixtures, were performed.

Table 1: Composition of the different systems used for simulations. The percentages indicate the solvent concentrations. The simulation $N^{o} 7$ was performed by using as starting conformation the AXT clusters obtained at the end of the simulation $N^{o} 6$.

\begin{tabular}{|c|c|c|c|c|c|c|}
\hline Sim. & $\begin{array}{c}\mathbf{N}^{o} \text { of AXT } \\
\text { molecules }\end{array}$ & $\begin{array}{c}\mathbf{N}^{o} \text { of Ethanol } \\
\text { molecules }\end{array}$ & $\begin{array}{c}\mathbf{N}^{o} \text { of water } \\
\text { molecules }\end{array}$ & Ethanol & \%water & $\begin{array}{c}\text { Box Size } \\
(\mathrm{nm})\end{array}$ \\
\hline 1 & 1 & 0 & 4000 & 0 & 100 & 6 \\
\hline 2 & 1 & 1923 & 0 & 100 & 0 & 6 \\
\hline \hline 3 & 10 & 464 & 20710 & 6 & 94 & 8.78 \\
\hline 4 & 10 & 2000 & 15662 & 28 & 72 & 12 \\
\hline 5 & 10 & 5000 & 15662 & 49 & 51 & 9.8 \\
\hline 6 & 10 & 10436 & 10477 & 75 & 25 & 10.96 \\
\hline 7 & 10 & 16715 & 1671 & 97 & 3 & 11.9 \\
\hline \hline 8 & 10 & 16715 & 0 & 100 & 0 & 11.9 \\
\hline
\end{tabular}

Translational and rotational coefficients. The translational self-diffusion coefficient $\left(D_{T}\right)$ of AXT was calculated from the mean square displacement of the center of mass of the molecules using the Einstein relation. ${ }^{33}$ To account for the differences between the transport properties of the solvent model with the experimental values, dynamics viscosities for both water and ethanol models were calculated using the non-equilibrium method developed by Hess, et al. ${ }^{34}$ Following this method, an external shear stress acceleration field was applied to the system in order to induce a velocity gradient with the same shape as the external acceleration field. The dynamic viscosity $(\eta)$ was derived from the resulting velocity using the same procedure described elsewhere. ${ }^{35}$

Finally, the viscosity corrected self-diffusion coefficients $\left(D_{T c}\right)$ were calculated from the Stokes-Einstein equation ${ }^{36}$ as follows: 


$$
D_{T c}=D_{T} \frac{\eta_{m}}{\eta_{\exp }}
$$

where $\eta_{m}$ and $\eta_{\text {exp }}$ are the model and experimental solvent viscosity.

The values of rotational relaxation time constants $(\tau)$ were estimated using the autocorrelation function of the unit vector $\mathbf{n}$ connecting the two carbon atoms at the beginning and the end of the polyene chain:

$$
C_{2}=\left\langle P_{2}(\mathbf{n}(0) \cdot \mathbf{n}(t))\right\rangle
$$

where the subscript 2 indicates that the correlation is calculated using the second order Legendre polynomial $P_{2}$ and the brackets \langle\rangle indicate the average along the trajectory. ${ }^{33}$ The value of the rotational relaxation time constants were estimated by fitting $C_{2}$ with a single exponential function. ${ }^{37}$ Also in this case, the values were corrected for the viscosity of the solvent as follows :

$$
\tau_{c}=\frac{\eta_{\exp }}{\eta_{m}} \tau
$$

The $D_{T c}$ and $\tau_{c}$ values were compared with those calculated using the program HYDROPRO. ${ }^{38,39}$ This program calculates hydrodynamic coefficients and other solution properties of molecules in the rigid body approximation. HYDROPRO was also used to estimate rotational diffusion coefficient $\left(D_{R}\right)$. To take into account the possible effect of AXT structural variations, the calculation was repeated on 2000 conformations sampled every 100 ps from the pure water and ethanol trajectories, and a statistical analysis was performed on the calculated values.

Calculation of the spatial density distribution. The spatial density 
function (SDF) distribution ${ }^{40}$ of the solvent molecules around the AXT molecule was calculated using the tool spatial of the GROMACS package using the last $5 \mathrm{~ns}$ of the simulations. Upon translational and rotational fitting of the systems to a reference AXT molecule, the atomic positions of selected solvent atoms (the oxygen in both solvent and the methyl group of the ethanol) were mapped on to a cubic grid with a grid spacing of $0.1 \mathrm{~nm}$ centred on the reference molecule. Hence, the grid densities have been averaged over the number of analyzed frames. Finally, The SDF have been inspected and analyzed using the program VMD. ${ }^{41}$

Analysis of AXT aggregates. The number of AXT molecules in an aggregate was calculated using an in-house written program. For each AXT conformation along the MD trajectory, the program built a contact matrix by counting the number contacts (defined as an interatomic distance $\leq 0.6 \mathrm{~nm}$ ) among the not hydrogen atoms of the AXT molecules. The contact matrix was then used to identify molecular clusters using the following method. For each molecule, neighbour molecules with a number of contacts larger than 250 were identified. The structure with the highest number of neighbours formed together with all its neighbours a (first) cluster. The search was then repeated in the same way until the pool of structures was empty. The final set of identified cluster represent the different isolated AXT aggregates present in each simulation frame. The relative orientations of the AXT molecules in each aggregate were evaluated by calculating the angle between the largest principal axis of each pair of AXT molecule.

The local ethanol concentration (LEC) around the AXT aggregates was obtained from the average cumulative number $\left(N_{\{w, e\}}\right)$ of water and ethanol molecules around the oxygen atoms of the AXT, calculated in the last 5 ns of the simula- 
tions, using the formula:

$$
L E C(r)=100 \frac{\bar{V}_{e} N_{e}}{\bar{V}_{e} N_{e}+\bar{V}_{w} N_{w}} .
$$

\section{Results and Discussion}

\section{Structural organisation of pure solvents around AXT}

The average SDFs around the AXT molecule in both water and ethanol simulations is shown on the right side of Fig. 2. For both the solvents, the regions with higher solvent density are localized in the proximity of the two terminal ring groups where, the presence of hydroxyl substituents, lead to the formation of stabilising hydrogen bond interactions. In the case of ethanol, these stabilising hydrogen bonds interactions cause the observed high densities (in grey) of methyl groups on both sides of the terminal rings (Fig.2b). In both the solvents, a low density is observed along the hydrophobic polyene chain.

The radial distribution functions (RDF) between oxygens of the AXT and those of two solvents (Fig.2, left graph) is in agreement with SDFs distributions showing a well defined first minimum (corresponding to the fist solvation shell) at 0.36 and $0.38 \mathrm{~nm}$ for water and ethanol, respectively. The first peaks is higher for ethanol than for water as a consequence of the different number of coordinating molecules. In fact, the averaged cumulative number of coordination molecules (Fig.2, left graph, dashed curves) calculated at the first minimum gives a number of water molecules (3.3) two times larger than the ethanol ones (1.7). The number molecules in the second coordination shell, calculated at the second minimum of the RDFs, are 15.8 (at 0.57$)$ and $4.9(0.59 \mathrm{~nm})$ for water and ethanol, respectively. The larger number of coordinating water molecules is likely a consequence of the 
size of the molecule that promote the formation of compact and stable clathratelike hydrogen bonding networks around the hydrophilic ring groups of the AXT.
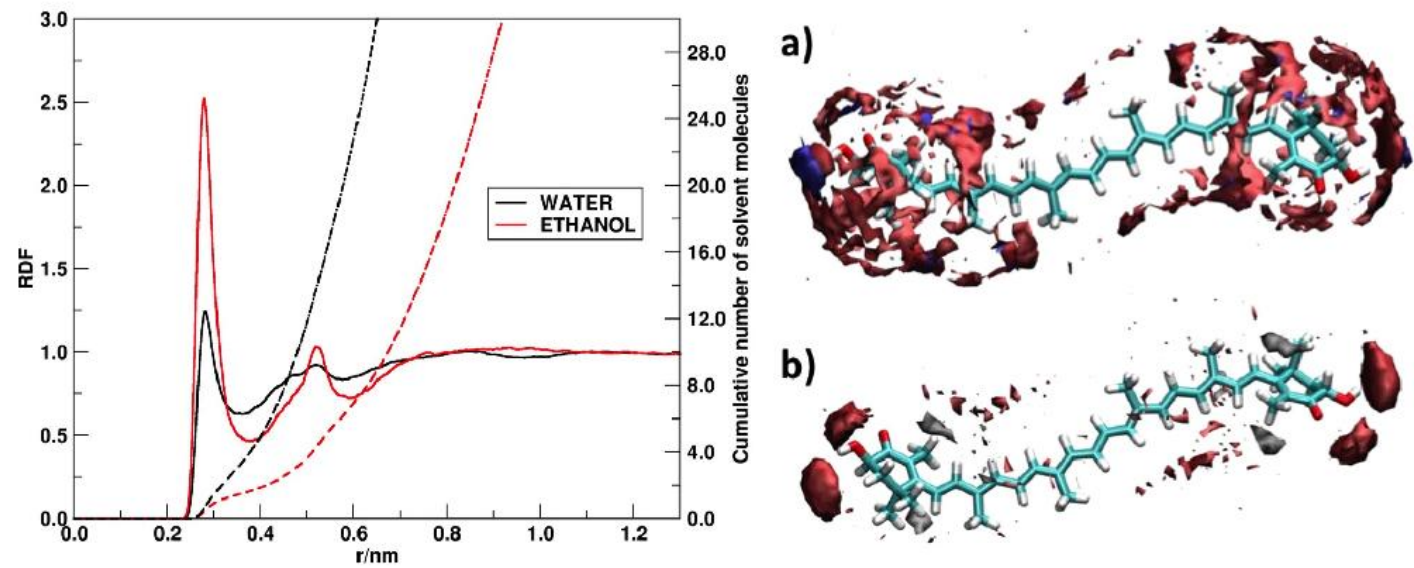

Figure 2: Left: RDF functions (solid lines) and average coordination number (dashed lines) for the hydroxyl $\mathrm{O}$ atoms of AXT with the $\mathrm{O}$ atoms of different solvents. Right: SDF of a) water (O in red, $\mathrm{H}$ in blue) and, b) ethanol unitedatom model $\left(\mathrm{C}_{2} \mathrm{H}_{5}\right)$ in grey, $\mathrm{OH}$ in red. The iso-surfaces are calculated at similar contour density values for both water and ethanol.

Translation and Rotation Diffusion coefficients. The values of $D_{T}$ for AXT in pure water and in ethanol are $(3.22 \pm 0.01) \times 10^{-6} \mathrm{~cm}^{2} \mathrm{~s}^{-1}$ and $(2.7 \pm 0.4) \times 10^{-6} \mathrm{~cm}^{2} \mathrm{~s}^{-1}$, respectively. The calculated values of viscosity of the water, and ethanol models are $\eta_{w}=(5.6 \pm 0.7) \times 10^{-4} \mathrm{~Pa} \cdot \mathrm{s}$, and $\eta_{e}=(7.4 \pm 0.9) \times 10^{-4}$ Pa.s, respectively. The ratio with the corresponding experimental values of $8.9 \times 10^{-4} \mathrm{~Pa} \cdot \mathrm{s}$ and $10.9 \times 10^{-4} \mathrm{~Pa} \cdot \mathrm{s}$, at the same temperature, give the diffusion correction factors of 0.63 and 0.68 , for water and ethanol, respectively. Using Equ. 1 , the corrected $D_{T c}$ values of $(2.03 \pm 0.01) \times 10^{-6} \mathrm{~cm}^{2} \mathrm{~s}^{-1}$ and $(1.8 \pm 0.3) \times 10^{-6}$ $\mathrm{cm}^{2} \mathrm{~s}^{-1}$ are obtained, respectively. These values are in excellent agreement with those of $(2.01 \pm 0.01) \times 10^{-6} \mathrm{~cm}^{2} \mathrm{~s}^{-1}$ and $(1.83 \pm 0.3) \times 10^{-6} \mathrm{~cm}^{2} \mathrm{~s}^{-1}$, for water and ethanol respectively, estimated from the AXT conformations using the HYDROPRO program. Unfortunately, to the best of our knowledge, experimental 
values of $D_{T}$ in water and ethanol are not yet available in literature. However, our calculated values in ethanol are of the same order of magnitude to those recently measured for dye molecules of comparable size and shape, ${ }^{42}$ making us confident that they are a good estimation of the experimental ones.

The viscosity corrected values of $\tau_{R}$ obtained from the ACF (see Methods) are equal to $3.9 \times 10^{9} \mathrm{~s}^{-1}$ and $4.5 \times 10^{9} \mathrm{~s}^{-1}$ in ethanol and in water, respectively. Again, these values are in quite good agreement with those calculated by HYDROPRO, which are $(2.8 \pm 0.4) \times 10^{9} \mathrm{~s}^{-1}$ and $(3.7 \pm 0.8) \times 10^{9} \mathrm{~s}^{-1}$, respectively. The values of $D_{R}$ as calculated by HYDROPRO from $\tau_{R}$ are $(1.4 \pm 0.1) \times 10^{7} \mathrm{~s}^{-1}$ and $(1.2 \pm 0.1) \times 10^{7} \mathrm{~s}^{-1}$ in water and ethanol, respectively. Also for these values, experimental data for a comparison are not yet available for this molecule.

Astaxanthin aggregation. The graphs on the left and middle columns in Fig.3 show the number of aggregates and the maximum number of molecules per cluster during the simulations, respectively. The structure of aggregates at the end of the simulations are shown on the right side of the Fig. 3. In mixtures, with water concentration $\leq 25 \%$, AXT molecules remain mostly dispersed as single molecules. Although also dimers (at 75\% and 95\% ethanol concentrations) and trimers (at 75\%) are also observed. With the increase in the concentration of water, the AXT molecules tend to minimize the exposure to the solvent of their hydrophobic regions by clumping together in larger compact clusters containing up to 8 molecules (see Fig. 3).

Fig. 4 shows a detailed view of the structure of aggregates at the end of the $5 \%, 25 \%$ and $50 \%$ ethanol/water mixtures simulations, respectively. In $50 \%$ ethanol (a), AXT molecules form a loose three-dimensional arrangement in which it is possible to identify a dimer, a trimer and several monomers (indicated by the arrows in the Figure). By decreasing the ethanol concentration (b), the ag- 

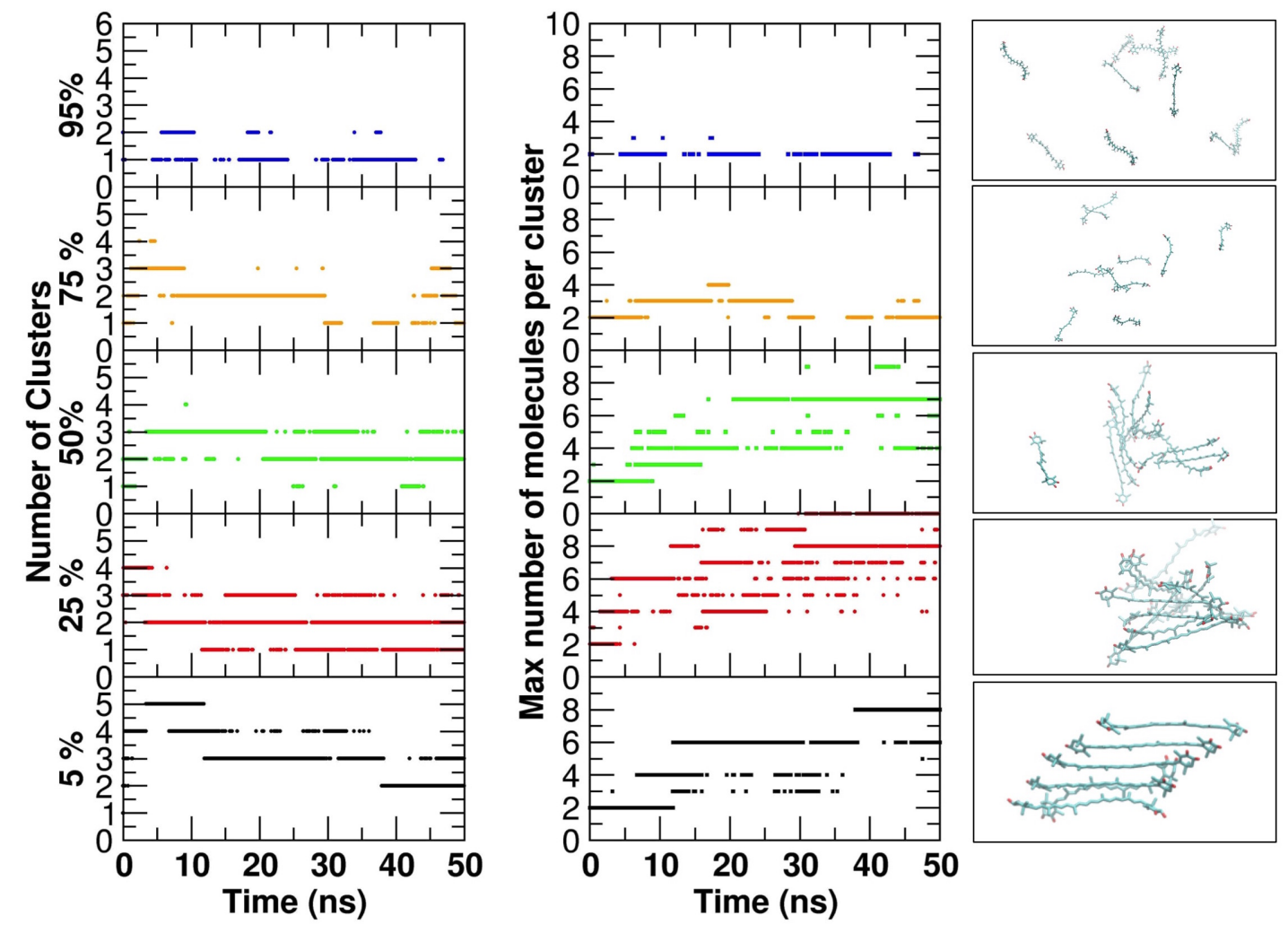

Figure 3: Aggregation dynamics of Astaxanthin molecules in the different mixture simulations. From the left to the right columns, number of molecular clusters and maximum number of molecules in the clusters are shown. On the right side, representations of the structure of AXT aggregates at the end of the simulations are shown. Concentrations are expressed in $\%(\mathrm{v} / \mathrm{v})$ of ethanol. 
gregate becomes more compact with a tubular shaped morphology. Some of the molecules, pointed out in the Figure by arrows, are arranged in a card-pack geometry while others are randomly oriented. The molecules in the dimer and the trimer are both arranged in a card-pack geometry. Finally, at the lowest ethanol concentration (c), 5\%, the molecules are stacked in groups of three dimers, indicated in the Fig. 4 by the labels i, ii and iii, respectively. The two monomers of each dimer are in card-pack arrangement while the dimers themselves are arranged in a head-to-tail configuration. The total slippage (defined as the average angle formed by the line through the center of the monomers when they are aligned with an horizontal axis ${ }^{43}$ ) of the aggregate is $\approx 50-55^{\circ}$. Large slippage $\left(32^{\circ}\right)$ are associated with hypsochromic shift of the absorption (H-type aggregation). ${ }^{43}$ As expected, the increase of the water concentration in the mixture affect both the number and the morphology of aggregates changing them from disordered and less compact three-dimensional assemblies to a more ordered two dimensional array. Interestingly, in our simulation the $2 \mathrm{~d}$ array has a slippage of $\geq 40^{\circ}$, which is the value observed in the crystallographic structure of the molecule ( $4 \mathrm{~d}$ ) crystallised from chloroform/hexane. ${ }^{44}$ The geometry of our aggregate also resemble those present in mixtures of water with $\leq 20 \%$ of acetone, ${ }^{45}$ and, for the analogous carotenoid zeaxanthin, in water with $\leq 20 \%$ of ethanol. ${ }^{46}$

Although the hydrophobic interactions play major role in the aggregation, the packed arrangements, as the one shown in Fig. 4(c), a network of hydrogen bonds between the bridging water molecules and $-\mathrm{OH}$ and $=\mathrm{O}$ groups in the terminal ring groups of AXT molecules could also play a role in the alignment of the AXT molecules. An example of such a network of hydrogen bonds in shown on the right side panel of Fig. 5. As shown in the LEC graph of Fig. 5), ethanol molecules tends to accumulate on the surface of the aggregates. The 

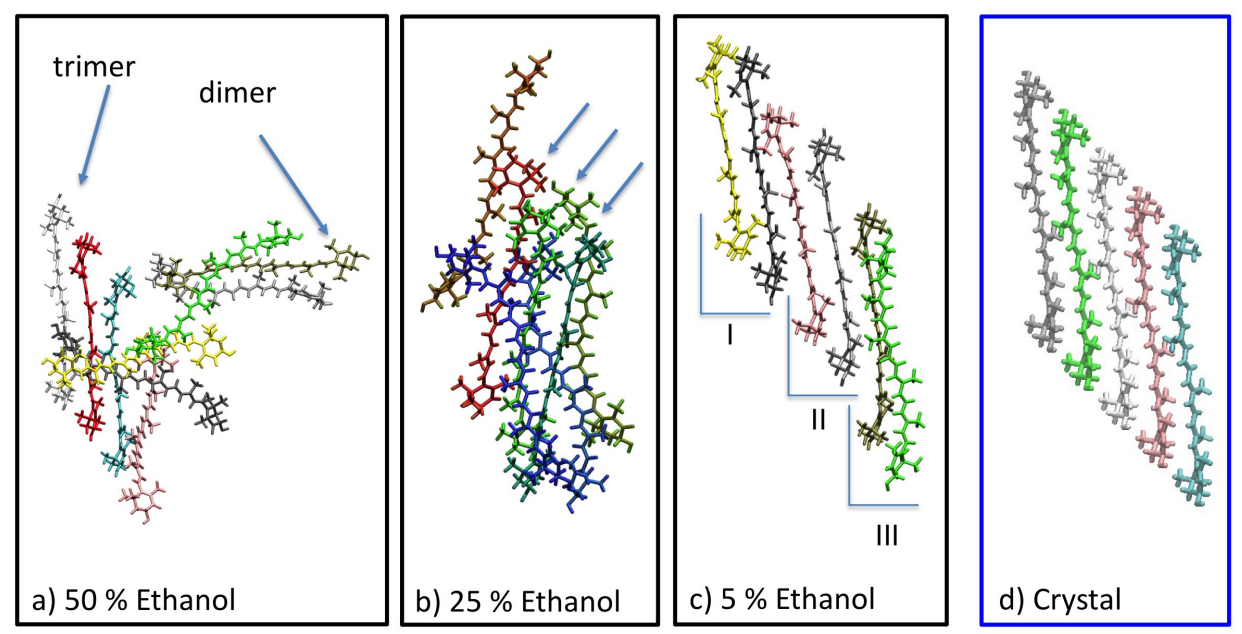

Figure 4: Representative structures of aggregates in ethanol mixtures with $50 \%$ (a), $25 \%$ (b) and $5 \%$ (c) of ethanol in the mixtures. In (d) the structure of the AXT in the crystal is reported.

LEC around the oxygen atoms of the AXT molecules in the different mixtures are characterised by ethanol concentrations higher than bulk phase with a maxima at $\approx 0.6 \mathrm{~nm}$ followed by a progressive decrease and convergence to the bulk value. This effect is particularly evident at low ethanol concentrations where LEC can rise 2-3 times higher than the bulk value (see Fig. 5).
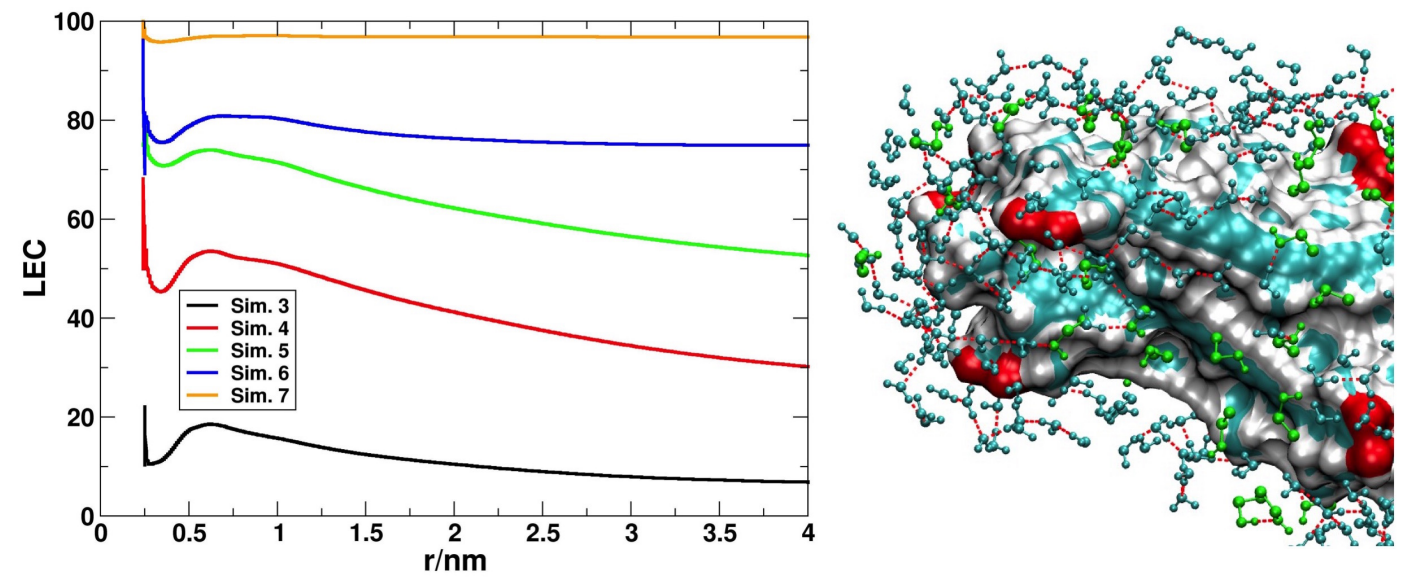

Figure 5: On the left, local ethanol concentration around AXT oxygens as function of the distance. On the right, an example of hydrogen bond network around the AXT aggregate at the end of the . 
Astaxanthin dissolution. In order to verify the reversibility of the aggregation, the cluster formed at the end of the $75 \%$ water/ethanol simulation was solvated in a new box of pure ethanol and simulated for $50 \mathrm{~ns}$. The bottom graphs of Fig.6, shows the evolution of the aggregate during the simulation. The initial cluster splits into smaller clusters that continue to progressively disaggregate to the single monomers within $50 \mathrm{~ns}$. The dissolving mechanism fit approximately a bi-exponential (Fig.6,bottom graph) with a very fast initial disaggregation phase $\left(\tau_{1} \approx 0.4 \mathrm{~ns}\right)$ followed by a much slower one $\left(\tau_{2} \approx 40 \mathrm{~ns}\right)$.
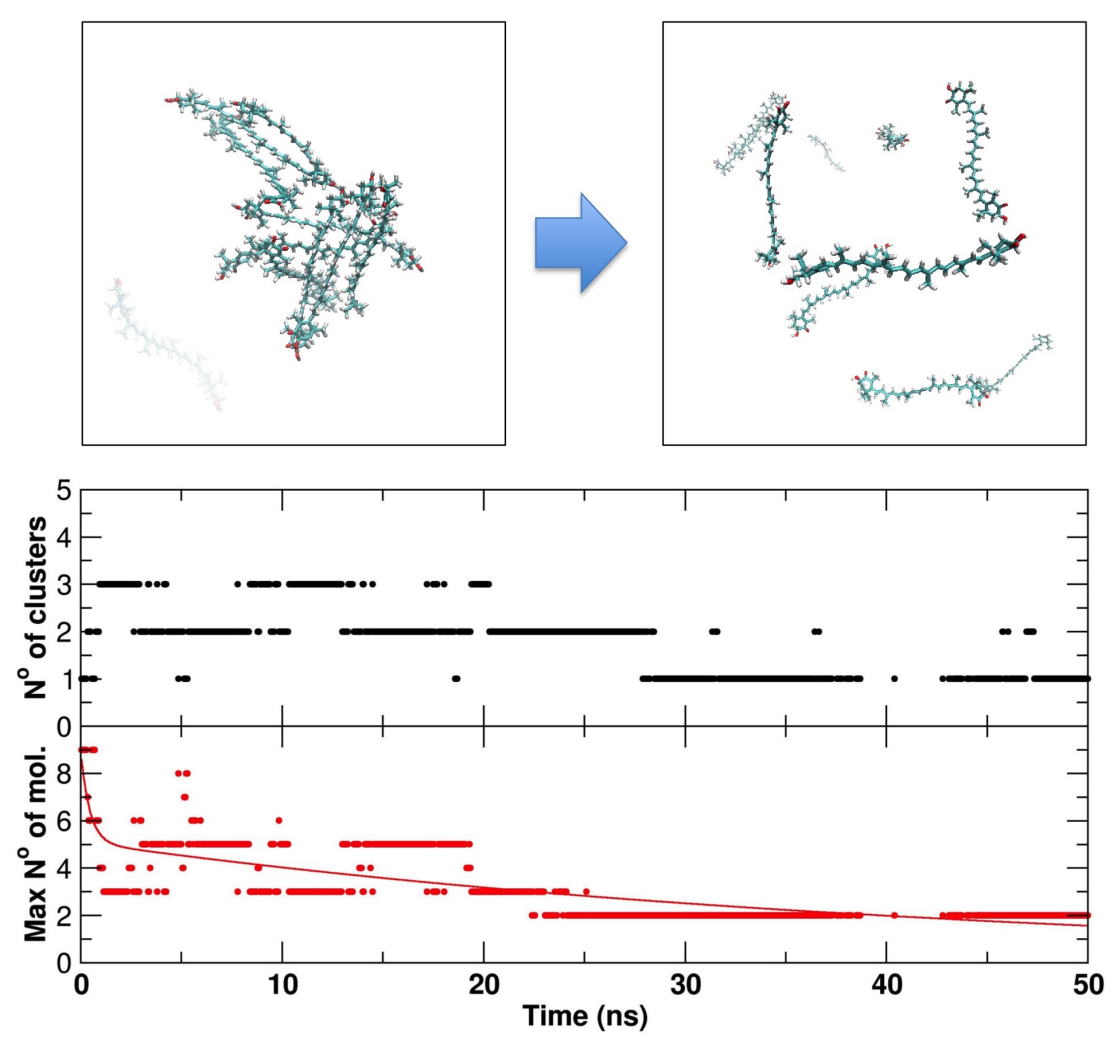

Figure 6: Dissolution of the aggregate obtained at the end of the simulation 5 (49\% (v/v) of ethanol) in pure ethanol. Top: the initial (left) and final configurations (right) after $50 \mathrm{~ns}$ of Sim 8. Bottom: the graphs of the number of clusters and maximum number of molecules in the aggregates are reported, respectively. 


\section{Conclusions}

We have presented a new molecular dynamics model of AXT based on the OPLS force field. Using the model we have calculated the structural and dynamic properties of AXT in water and ethanol solutions. We have also used the model to perform a preliminary study on the aggregation of AXT molecules in the mixtures of the two solvents. The simulations show that the amount of water present in the mixtures changes the morphology of the aggregates which qualitatively agree with the experimental data For water contents below $50 \%$ (v/v), small threedimensional aggregates of dimers/trimers that are either isolated or loosely connected are observed. As the water content increases to $75 \%$ (v/v), the aggregates are arranged in a compact cylindrical three-dimensional structure. Finally, at the highest water content $(>95 \%)$, the molecules tend to form two-dimensional arrays that resemble the crystal structure. However, the three-dimensional aggregates cannot be unambiguously identified as $H$ or $J$-aggregates. This aspect can be analysed in a future studies on the spectroscopic properties of the aggregates generated by our model. We also observe that although hydrophobic interactions play major role in aggregation, a network of hydrogen bonds between the -OH and $=\mathrm{O}$ groups of AXT and water molecules play important role in the directed arrangement of the AXT molecules. Aggregation is not observed in mixtures in which the water content is lower than $50 \%$.

Aggregation of organic molecules in solution, in particular molecules with pharmacological or nutritive properties, is an important research topics. In fact, recent studies have shown that aggregation is quite common phenomena in hydrophobic drugs (as e.g anticancer drugs), and their aggregation in-vivo can

strongly affect their efficacy ${ }^{18,19}$ Therefore, this study contributes to the general 
understanding of the mechanism of natural product aggregation.

We are currently working to study the aggregation properties of AXT in different solvents and to investigate more quantitatively the kinetics of aggregation and dissolution. As the model is compatible with the OPLS force-field, it can also be used to investigate the interaction of AXT molecules with proteins and the effects of aggregation on its functionality. In particular, it will be interesting to study the association of the AXT with its natural binding protein in the crustacyanin whose crystallographic structures have been recently solved. ${ }^{47}$

\section{Supporting Information Available}

Details of the optimized structure of Astaxanthin, and force field parameters (including details of all the bonded and nonbonded parameters) are available in the Supporting Information.

This material is available free of charge via the Internet at http://pubs acs. org/.

\section{Acknowledgement}

The authors thank IASTE student trainee Miss Justyna Lenda for her help at the beginning of this project. Part of this study was performed using the computational resources of the CLAMV (Computer Laboratories for Animation, Modeling and Visualization) at Jacobs University Bremen. 


\section{References}

(1) Miki, W. Biological functions and activities of animal carotenoids. Pure \& Appl. Chem. 1991, 63, 141-146.

(2) Wayama, M.; Ota, S.; Matsuura, H.; Nango, N.; Hirata, A.; Kawano, S. Three-dimensional ultrastructural study of oil and astaxanthin accumulation during encystment in the green alga Haematococcus pluvialis. PloS one 2013, 8, e53618.

(3) Mortensen, A.; Skibsted, L. H. Importance of Carotenoid Structure in Radical-Scavenging Reactions. J. Agric. Food Chem. 1997, 45, 2970-2977.

(4) Goswami, G.; Chaudhuri, S.; Dutta, D. The present perspective of astaxanthin with reference to biosynthesis and pharmacological importance. World J. Microbiol. Biotechnol. 2010, 26, 1925-1939.

(5) Hussein, G.; Sankawa, U.; Goto, H.; Matsumoto, K.; Watanabe, H. Astaxanthin, a carotenoid with potential in Human Health and Nutrition. J. Nat. Prod. 2006, 69, 443-449.

(6) Yoshihisa, Y.; Rehman, M. U.; Shimizu, T. Astaxanthin, a xanthophyll carotenoid, inhibits ultraviolet-induced apoptosis in keratinocytes. Exp. Dermatol. 2014, 23, 178-183.

(7) Fuciman, M.; Durchan, M.; Šlouf, V.; Keşan, G.; Polívka, T. Excited-state dynamics of astaxanthin aggregates. Chem. Phys. Lett. 2013, 568-569, 2125.

(8) Musser, A. J.; Maiuri, M.; Brida, D.; Cerullo, G.; Friend, R. H.; Clark, J. 
The Nature of Singlet Exciton Fission in Carotenoid Aggregates. Journal of the American Chemical Society 2015, 137, 5130-5139.

(9) Ballschmiter, K.; Katz, J. J. An Infrared Study of Chlorophyll-Chlorophyll and Chlorophyll-Water Interactions. J. Am. Chem. Soc. 1969, 91, 26612677.

(10) Katz, J. J.; Norris, J. R.; Shipman, L. L.; Thurnauer, M. C. Chlorophyll Function in the Photosynthetic Reaction Center. Ann. Rev. Biophys. Bioeng. 1978, 7, 393-434.

(11) Karki, K.; Roccatano, D. Molecular Dynamics Simulation Study of Chlorophyll a in Different Organic Solvents. J. Chem. Theory. Comput. 2011, 7, $1131-1140$.

(12) Britton, G., Liaaen-Jensen, S., Pfander, H., Eds. Carotenoids, Volume 4: Natural Functions; Birkhäuser Verlag: Basel, 2008.

(13) Köpsel, C.; Möltgen, H.; Schuch, H.; Auweter, H.; Kleinermanns, K.; Martin, H.-D.; Bettermann, H. Structure investigations on assembled astaxanthin molecules. J. Mol. Struct. 2005, 750, 109-115.

(14) Giovannetti, R.; Alibabaei, L.; Pucciarelli, F. Kinetic model for astaxanthin aggregation in water-methanol mixtures. Spectrochim Acta A 2009, 73, 157162.

(15) Calogero, G.; Bartolotta, A.; Di Marco, G.; Di Carlo, A.; Bonaccorso, F. Vegetable-based dye-sensitized solar cells. Chemical Society Reviews 2015, 44, 3244-3294. 
(16) Spano, F. C. Analysis of the UV/Vis and CD spectral line shapes of carotenoid assemblies: Spectral signatures of chiral $H$-aggregates. J. Am. Chem. Soc. 2009, 131, 4267-4278.

(17) Jorgensen, W. L. Encyclopedia of Computational Chemistry; Wiley: New York, 1998; Vol. 3; Chapter OPLS, force field, pp 1986-1989.

(18) Owen, S. C.; Doak, A. K.; Wassam, P.; Shoichet, M. S.; Shoichet, B. K. Colloidal aggregation affects the efficacy of anticancer drugs in cell culture. ACS Chem. Biol. 2012, 7, 1429-1435.

(19) Sassano, M. F.; Doak, A. K.; Roth, B. L.; Schoichet, B. K. Colloidal aggregation causes inhibition of $\mathrm{G}$ protein-coupled receptors. J. Med. Chem. 2013, 56, 2406-2414.

(20) Frisch, M. J. et al. Gaussian 09 Revision A.1. Gaussian Inc. Wallingford CT 2009 .

(21) Breneman, C. M.; Wiberg, K. B. J. Comp. Chem. 1990, 11, 361-397.

(22) Jorgensen, W. L.; Tiraldo-Rives, J. The OPLS [optimized potentials for liquid simulations] potential functions for proteins, energy minimizations for crystals of cyclic peptides and crambin. J. Am. Chem. Soc. 1988, 110, $1657-1666$.

(23) Jorgensen, W. L.; Maxwell, D. S.; Tiraldo-Rives, J. Development and testing of the OPLS all-atom force field on conformational energetics and properties of organic liquids. J. Am. Chem. Soc. 1996, 118, 11225-11236.

(24) Jorgensen, W. L.; McDonald, N. A. Development of an All-Atom Force Field 
for Heterocycles. Properties of Liquid Pyridine and Diazenes. J. Mol. Struct. (THEOCHEM) 1998, 424, 145-155.

(25) Jorgensen, W. L.; McDonald, N. A. Development of an All-Atom Force Field for Heterocycles. Properties of Liquid Pyrrole, Furan, Diazoles, and Oxazoles. J. Phys. Chem. B 1998, 102, 8049-8059.

(26) Jorgensen, W. L.; Maxwell, D. S.; Tirado-Rives, J. Development and Testing of the OPLS All-Atom Force Field on Conformational Energetics and Properties of Organic Liquids. J. Am. Chem. Soc. 1996, 118, 11225-11236.

(27) Berendsen, H. J. C.; Grigera, J. R.; Straatsma, T. P. The missing term in effective pair potentials. J. Phys. Chem. 1987, 91, 6269-6271.

(28) Hess, B.; Kutzner, C.; van der Spoel, D.; Lindahl, E. GROMACS 4: Algorithms for highly efficient, load-balanced and scalable molecular simulation. J. Chem. Theory Comput. 2008, 4, 435-447.

(29) Hoover, W. G. Canonical dynamics: equilibrium phase-space distributions. Phys. Rev. A 1985, 31, 1695-1697.

(30) Parrinello, M.; Rahman, A. Polymorphic transitions in single crystals: A new molecular dynamics method. J. Apply. Phys. 1981, 52, 7182-7190.

(31) Ryckaert, J.-P.; Ciccotti, G.; Berendsen, H. J. C. Numerical Integration of the Cartesian Equations of Motion of a System with Constraints: Molecular Dynamics of n-Alkanes. J. Comput. Chem. 1977, 23, 327-341.

(32) Essmann, U.; Perera, L.; Berkowitz, M. L.; Darden, T.; Lee, H.; Pedersen, L. G. A smooth particle mesh ewald potential. J. Chem. Phys. 1995, 103, 8577-8592. 
(33) Allen, M. P.; Tildesley, D. J. Computer Simulations of Liquies; Oxford Science Publications: Oxford, 1987; Chapter Statistical Mechanics, pp 5860.

(34) Hess, B. Determining the Shear Viscosity of Model Liquids From Molecular Dynamics. J. Chem. Phys. 2002, 116, 209-217.

(35) Hezaveh, S.; Samanta, S.; Milano, G.; Roccatano, D. Structure and dynamics of 1,2-dimethoxyethane and 1,2-dimethoxypropane in aqueous and non-aqueous solutions: A molecular dynamics study. The Journal of Chemical Physics 2011, 135, 164501-164510.

(36) Berg, H. C. Random walks in biology; Princeton University Press, 1993.

(37) Jas, G. S.; Wang, Y.; Pauls, S. W.; Johnson, C. K.; Kuczera, K. Influence of Temperature and Viscosity on Anthracene Rotational Diffusion in Organic Solvents: Molecular Dynamics Simulations and Fluorescence Anisotropy Study. J. Chem. Phys. 1997, 107, 8800-8812.

(38) Carrasco, B.; de la Torre, J. G. Hydrodynamic properties of rigid particles: Comparison of different modeling and computational procedures. Biophys. J. 1999, 76, 3044-3057.

(39) Ortega, A.; Amoros, D.; de la Torre, J. G. Prediction of hydrodynamic and other solution properties of rigid proteins from atomic- and residue-level models. Biophys. J. 2011, 101, 892-898.

(40) Kulasik, P. G.; Laaksonen, A.; Svishchev, I. M. Molecular dynamics: from classical to quantum methods; Elsevier Science B.V.: Sara Burgerhartstraat 25, Amsterdam, 1999; Chapter Spatial Structure in Molecular Liquids, pp $61-90$. 
(41) Humphrey, W.; Dalke, A.; Schulten, K. VMD: Visual molecular dynamics. J. Mol. Graphics 1996, 14, 33-38.

(42) Goosens, K.; Prior, M.; Pacheco, V.; Willbold, D.; Müllen, K.; Enderlein, J.; Hofkens, J.; Gregor, I. Accurate diffusion coefficients of organosoluble reference dyes in organic media measured by dual-focus fluorescence correlation spectroscopy. ACS Nano 2015, 9, 7360-7373.

(43) Köhn, S.; Kolbe, H.; Korger, M.; Köpsel, C.; Mayer, B.; Auweter, H.; Lüddecke, E.; Bettermann, H.; Martin, H.-D. carotenoids; Springer, 2008; pp 53-98.

(44) Giuditta, B.; Jennifer, C.; Stuart, F.; Gillian, H.; Helliwell, J. R.; Helliwell, M.; Synnove, L.-J. Acta Crystallogr., Sect. B 2007, 63, 328-337.

(45) Köpsel, C.; Möltgen, H.; Schuch, H.; Auweter, H.; Kleinermanns, K.; Martin, H.-D.; Bettermann, H. Structure investigations on assembled astaxanthin molecules. Journal of molecular structure 2005, 750, 109-115.

(46) Billsten, H. H.; Sundström, V.; Polívka, T. Self-assembled aggregates of the carotenoid zeaxanthin: time-resolved study of excited states. The Journal of Physical Chemistry A 2005, 109, 1521-1529.

(47) Cianci, M.; Rizkallah, P. J.; Olczak, A.; Raftery, J.; Chayen, N. E.; Zagalsky, P. F.; Helliwell, J. R. The molecular basis of the coloration mechanism in lobster shell: $\beta$-crustacyanin at $3.2-\AA$ resolution. Proceedings of the $\mathrm{Na}$ tional Academy of Sciences 2002, 99, 9795-9800. 


\section{Graphical TOC Entry}

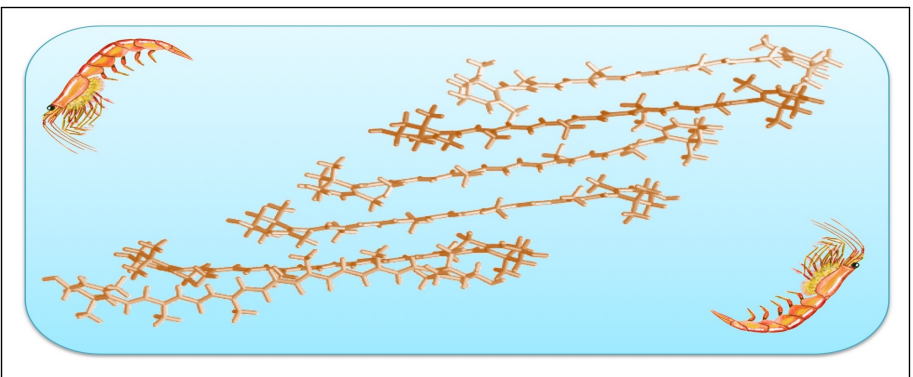

An example of molecular aggregate formed by Astaxanthin, an important carotenoid abundant in Antarctic krill, in aqueous ethanolic solutions. 